\title{
Efficiency limits of electronically-coupled upconverter and quantum ratchet solar cells using detailed balance
}

\author{
Emily Z. Zhang* \\ Department of Physics, University of Ottawa, Ottawa, ON, Canada \\ Jacob J. Krich \\ Department of Physics, University of Ottawa, Ottawa, ON, Canada and \\ School of Electrical Engineering and Computer Science, University of Ottawa, Ottawa, ON, Canada

\begin{abstract}
The intermediate band solar cell (IBSC) and quantum ratchet solar cell (QRSC) have the potential to surpass the efficiency of standard single-junction solar cells by allowing sub-gap photon absorption through states deep inside the band gap. High efficiency IBSC and QRSC devices have not yet been achieved, however, since introducing mid-gap states also increases recombination, which can harm the device. We consider the electronically coupled upconverter (ECUC) solar cell and show that it can achieve the same efficiencies as the QRSC. Although they are equivalent in the detailed balance limit, the ECUC is less sensitive to nonradiative processes, which makes it a more practical implementation for IB devices. We perform a case study of crystalline-silicon based ECUC cells, focusing on hydrogenated amorphous silicon as the upconverter material and highlighting potential
\end{abstract} \\ dopants for the ECUC. These results illustrate a new path for the development of IB-based devices.
}

\section{INTRODUCTION}

Shockley and Queisser used the detailed balance (DB) formalism to show that the efficiency of a solar cell made from a semiconductor with a single band gap can never exceed $31 \%$ under unconcentrated black-body sunlight [1. Intermediate band (IB) materials - semiconductors with allowed electronic states deep in the gap, as shown in Figure $1 \mathrm{a}$ - enable solar cells to break this limit by absorbing sub-gap photons without harming the voltage of the cell [2]. In the radiative limit, the maximum efficiency of an intermediate band solar cell (IBSC) at one sun concentration is $47 \%$, significantly exceeding the ShockleyQueisser limit 2]. Several intermediate band devices have been demonstrated, but high efficiencies have not been realized due to nonradiative recombination [3].

The quantum ratchet (QR) solar cell has been proposed as an improved implementation of the IBSC 4 . The intermediate band $\mathrm{QR}$ and conduction band QR implementations are shown in Figure 1 $1 \mathrm{~b}$-c, respectively. The original idea of a IBQR solar cell is to increase the lifetime of the IB. In the case of the IBQR, carriers relax from the IB to a ratchet band (RB), which can suppress recombination to the valence band (VB). The ratchet also enables improved voltage matching between the subgap transitions and the band-to-band transitions [5, 6]. The CBQR has the ratchet step above the conduction band edge, and an analogous valence band QR (not shown) has the ratchet step below the valence band edge. All three QR designs realize the voltage-matching improvements and can achieve detailed balance maximum efficiencies of $48.5 \%$ at one sun, greater than that of IBSCs. There have, however, been few $\mathrm{QR}$ experimental realizations

\footnotetext{
* Current address: Department of Physics, University of Toronto, Toronto, ON, Canada
}

and there are few suggestions for material systems [7].

In both IBSC and QRSC devices, the IB and QR regions are added to standard $p n$ junctions in hopes of increasing current in the device, but if lifetimes are sufficiently short in the IB region, the IBSC or QRSC may even have lower current than the reference $p n$ junction. Both IBSCs and QRSCs have an $n$-IB- $p$ architecture, implying the holes created at the front of the cell must travel through the IB region to be collected. If hole lifetimes in the IB or $\mathrm{QR}$ regions are short, the nonradiative losses in the IB region will exceed the extra current generation, making efficiencies less than for the $p n$-diode solar cell alone [8, 9].

The electronically-coupled upconverter (ECUC) is a less-studied architecture, which provides the potential to realize the same efficiency as a QRSC while being less sensitive to nonradiative processes [10, 11]. As shown in Figure 1 $1 \mathrm{~d}$, the ECUC has an $n$ - $p$-IB structure, with the IB region having a larger band gap than the standard semiconductor, unlike in the IBSC and QRSC where the large band gap $E_{C V}$ can be uniform through the device. As with IBSC and QRSC, the ECUC allows absorption of subgap photons, with the resulting carriers injected into the standard semiconductor. The minority carriers produced by absorption in the $p n$ junction never transit the IB region, so the current added from IB absorption can be obtained strictly as an addition, and low quality upconverter material cannot harm the cell as can occur in the IBSC/QRSC. However, the ECUC requires more complicated $2 \mathrm{D}$ contacts to avoid extracting current from the IB, with one possibility shown in Figure 2.

The detailed balance limiting efficiencies for the ECUC have not previously been calculated. In this work, we demonstrate that the QRSC and ECUC are mathematically equivalent in the DB limit, yet the ECUC may be a more practical implementation in actual devices. We show that, as with the QRSC, the ECUC configuration 

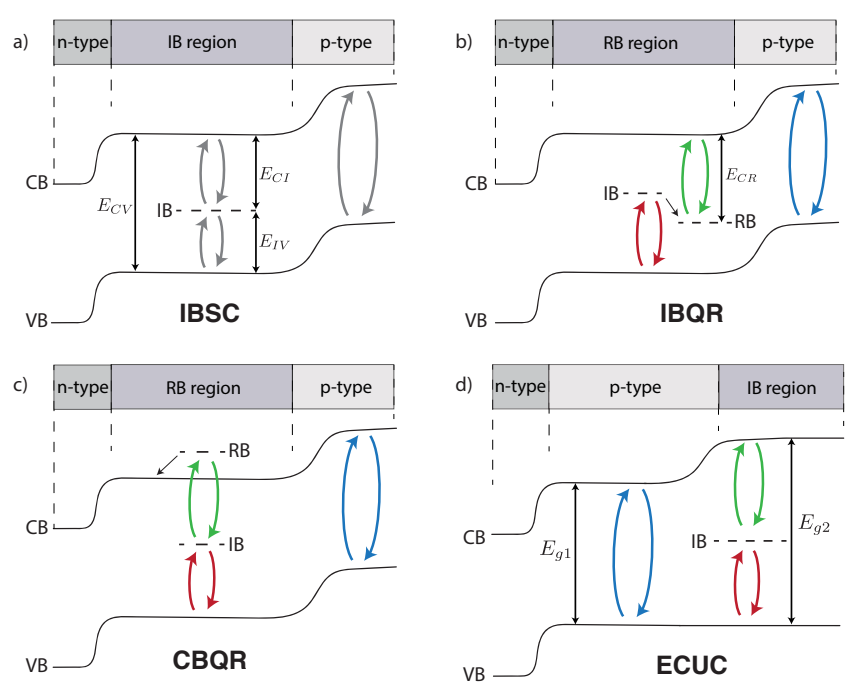

FIG. 1. Band diagrams of (a) IBSC, (b) IBQR, (c) CBQR, and (d) ECUC. The red, green, and blue processes for the ratchets and ECUC are equivalent in detailed balance.

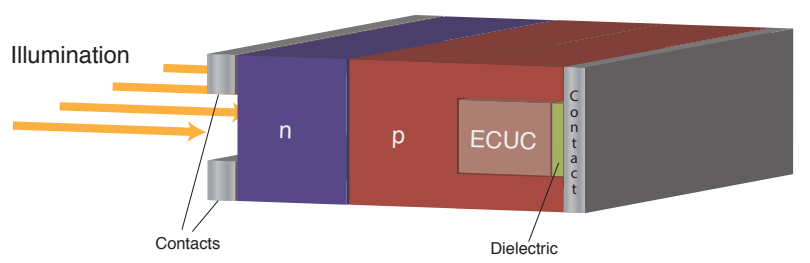

FIG. 2. Schematic of a potential device architecture for the ECUC.

has the potential to exceed IBSC efficiencies at 1 sun. We perform a global optimization showing the maximum efficiencies possible as functions of $E_{g 1}$ and $E_{g 2}$ and also consider a case study of an ECUC based on crystalline silicon (c-Si), the most widely used and studied PV material. We show that there is potential to improve on $\mathrm{c}-\mathrm{Si}$ solar cells using an ECUC.

\section{DETAILED BALANCE MODEL}

We use the well-known detailed balance formalism to model the ECUC and QRSC. We first show that in detailed balance, the ECUC and QRSC are mathematically equivalent, then we use this method to compute the limiting efficiencies for ECUC.

Detailed balance calculations assume all recombination is radiative, carriers have infinite mobility, and the cell is thick enough to assure full absorption of photons for each allowable transition. We further assume perfect photon selectivity, with each photon absorbed only by the highest-energy transition energetically permitted, to minimize thermalization losses; this condition is called non-overlapping absorptions and is not required for de- tailed balance [2, 12, 13]. Since the carriers have infinite mobility,

$$
\mu_{C V}=q V_{\text {ext }}
$$

where $q$ is the elementary charge, $\mu_{C V}$ is the quasi-Fermi level difference between the electrons and holes, and $V_{\text {ext }}$ is the external voltage. We take $q=1$.

Another key assumption is that there is one electron-hole pair generated/lost for each photon absorbed/emitted. Since all recombination events are assumed to be radiative, this assumption allows the current in the device to be written in terms of the photon fluxes $\phi$ in and out of the device. These fluxes obey the modified Planck spectrum 14

$$
\begin{aligned}
\phi\left(E_{\min , A B},\right. & \left.E_{\max , A B}, T, \mu_{A B}\right) \\
= & \frac{2 F}{h^{3} c^{2}} \int_{E_{\min , A B}}^{E_{\max , A B}} \frac{E^{2} d E}{e^{\left(E-\mu_{A B}\right) / k T}-1},
\end{aligned}
$$

where the process between bands $A$ and $B$ absorbs photons with energies between $E_{\min , A B}$ and $E_{\max , A B}, T$ is the temperature, $\mu_{A B}$ is the chemical potential difference between carriers in bands $A$ and $B, h$ is Planck's constant, $c$ is the speed of light, $k$ is Boltzmann's constant, and $F$ is the geometrical factor denoting the fraction of light incident on the cell. For the sun,

$$
F_{\text {sun }}=X \cdot \pi\left(\frac{\text { radius of sun }}{\text { distance between earth and sun }}\right)^{2},
$$

where $X$ is the solar concentration factor, and for emission from the cell,

$$
F_{\text {cell }}=\pi
$$

In detailed balance, we have two photon sources: the sun and the cell. We can denote the photons absorbed from the sun in transitions between bands $A, B$ by

$$
\dot{N}_{A B}^{\operatorname{sun}}=\phi\left(E_{\min , A B}, E_{\max , A B}, T_{s}, 0\right),
$$

and the photons emitted by the cell in transitions between bands $A, B$ by

$$
\dot{N}_{A B}^{\text {cell }}=\phi\left(E_{\min , A B}, E_{\max , A B}, T_{a}, \mu_{A B}\right),
$$

where $T_{s}$ is the solar radiation temperature, which we take to be $6000 \mathrm{~K}$ and $T_{a}$ is the ambient temperature, which we take to be $300 \mathrm{~K}$. The current extracted from band $A$ is the difference between absorbed and emitted photons involving band $A$,

$$
J_{A}=\sum_{B} \pm\left(\dot{N}_{A B}^{\text {sun }}-\dot{N}_{A B}^{\text {cell }}\left(\mu_{A B}\right)\right)
$$

with the sign depending on whether the $A B$ absorption process creates $(+)$ or destroys (-) carriers in band $A$. 
For all of the devices, the total current is the net current extracted from either the $\mathrm{CB}$ or the $\mathrm{VB}$, which are equal. For an ECUC, the total current is

$$
J_{C}^{E C U C}=\dot{N}_{C V}^{\mathrm{sun}}-\dot{N}_{C V}^{\mathrm{cell}}\left(\mu_{C V}\right)+\dot{N}_{C I}^{\mathrm{sun}}-\dot{N}_{C I}^{\mathrm{cell}}\left(\mu_{C I}\right) .
$$

We also assume that no current is extracted from the intermediate band, so

$$
J_{I}^{E C U C}=0=\dot{N}_{I V}^{\text {sun }}-\dot{N}_{I V}^{\text {cell }}\left(\mu_{I V}\right)-\dot{N}_{C I}^{\text {sun }}+\dot{N}_{C I}^{\text {cell }}\left(\mu_{C I}\right) .
$$

Note that the CI processes in Eq. 9 enter with the negative sign, as optical absorption from IB to CB removes an IB carrier. With equations 1899 , and the fact that

$$
\mu_{C V}=\mu_{C I}+\mu_{I V}
$$

we can solve for the chemical potentials and compute $J(V)$. These equations are of the same form as in the original IBSC calculation [2], but the ECUC has different band gaps in the different regions. Note that the $\mu_{C V}$ terms use $E_{g 1}$ as their lower threshold.

For an IBQR, we assume the carriers in the IB and $\mathrm{RB}$ share a common quasi-Fermi level, so $\mu_{C I}=\mu_{C R}$ [4]. Then, the net current from the CB is

$$
J_{C}^{I B Q R}=\dot{N}_{C V}^{\mathrm{sun}}-\dot{N}_{C V}^{\mathrm{cell}}\left(\mu_{C V}\right)+\dot{N}_{C R}^{\mathrm{sun}}-\dot{N}_{C R}^{\mathrm{cell}}\left(\mu_{C R}\right),
$$

and the net current in the IB is

$J_{I}^{I B Q R}=0=\dot{N}_{I V}^{\mathrm{sun}}-\dot{N}_{I V}^{\mathrm{cell}}\left(\mu_{C R}\right)-\dot{N}_{C R}^{\mathrm{sun}}+\dot{N}_{C R}^{\mathrm{cell}}\left(\mu_{C R}\right)$

These equations for the ECUC and IBQR are equivalent. As shown in Figure 11d, $E_{C I}+E_{I V}=E_{g 2}$ for the ECUC. If we choose $E_{C V}$ for the IBQR to equal $E_{g 1}$ for the ECUC then the first two terms in each of Eqs. 11 and 12 are equal to the equivalent terms in Eqs. 8 and 9. Further, if $E_{C R}$ for the IBQR equals $E_{C I}$ for the ECUC, and $E_{I V}+E_{C R}$ for the IBQR equals $E_{g 2}$ for the ECUC, then the last two terms in each of those equations become equivalent. Therefore the ECUC equations are equal to the IBQR equations. Similarly, if $E_{I V}+E_{R I}=E_{g 2}$ for the CBQR or $E_{I R}+E_{C I}=E_{g 2}$ for the valence band QR (VBQR), then the equations also become equivalent to the ECUC. Since the equations for QR and ECUC are no different in detailed balance, the limiting efficiencies are also the same.

Figure 3 shows the maximum ECUC efficiencies at $X=1$ and $X=1 / F_{\text {sun }}=46200$, which is the maximum value. The peak efficiencies and band gaps for these cases are shown in Table I. The diagonal border at $E_{g 1}=E_{g 2}$ represents standard IB solar cells, and at one sun concentration (left), the detailed balance efficiency is highest at $E_{g 1} \neq E_{g 2}$. This result indicates that the ECUC has higher limiting efficiency than IBSC, similar to QR [4], spectrally-selective reflectors [15], and overlapping absorptions 12. Therefore, the ECUC can exceed both the IBSC limit and the Shockley-Queisser
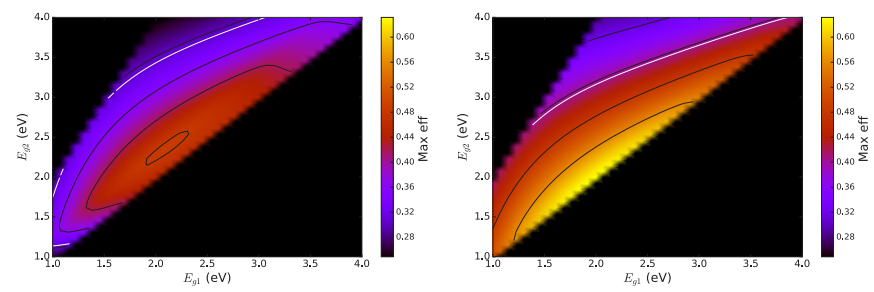

FIG. 3. Maximum ECUC efficiency in detailed balance, with optimized $E_{I}$, at 1 sun concentration (left) and at full concentration $(X=46200)$ (right). The Shockley-Queisser limits of $31 \%(X=1)$ and $40.7 \%(X=46200)$ are shown with the white contours. Note that an ECUC can only be beneficial if $E_{g 1} \leq E_{g 2} \leq 2 E_{g 1}$, with the second inequality from the requirement that both sub-gap transitions have energy thresholds below $E_{g 1}$.

TABLE I. Maximum efficiencies with a blackbody spectrum at 1 sun and full concentration. Note that there is a symmetry for $E_{I}$ mirrored below and above $E_{g 2} / 2$; we take the upper values for $E_{I}$.

\begin{tabular}{cccccc}
\hline System & $X$ & $E_{g 1}(\mathrm{eV})$ & $E_{g 2}(\mathrm{eV})$ & $E_{I}(\mathrm{eV})$ & Efficiency \\
\hline Single-junction & 1 & 1.31 & - & - & $31.0 \%$ \\
IB solar cell & 1 & 2.42 & - & 1.49 & $46.8 \%$ \\
ECUC solar cell & 1 & 2.08 & 2.36 & 1.42 & $48.5 \%$ \\
Single-junction & 46200 & 1.11 & - & - & $40.7 \%$ \\
IB solar cell & 46200 & 1.95 & - & 1.24 & $63.2 \%$ \\
ECUC solar cell & 46200 & 1.95 & 1.95 & 1.24 & $63.2 \%$ \\
\hline
\end{tabular}

limit. Figure 3 shows that there is a wide range of band gaps that can potentially achieve this goal.

At full concentration, the highest efficiency lies on $E_{g 1}=E_{g 2}$, so there is no gain from ECUC compared to a standard IBSC architecture. Both the ECUC and the IBSC significantly exceed the single junction efficiency limit, which has motivated interest in combining IBSC with concentrator systems [16, 17.

\section{CASE STUDY: ECUC USING C-SI}

In this section, we perform a case study of a potential ECUC using silicon as the front $p n$-diode material, since c-Si is an extremely well-understood material. Adding only an intermediate band to an $n$-IB- $p$ c-Si solar cell actually harms the efficiency of the cell, even in the detailed balance limit 12 . That failure occurs because of silicon's small band gap and the assumption of non-overlapping absorptions. Figure 3, however, shows that even with $E_{g 1}$ equal to the band gap of c-Si, 1.12 $\mathrm{eV}$, the ECUC allows considerable improvement over the Shockley-Queisser limit. First, we study the optimal range for $E_{g 2}$ for an ECUC on silicon. Second, we consider an ECUC made of hydrogenated amorphous silicon (a-Si), which is a higher band-gap material frequently used for heterojunctions with c-Si. We perform a search 


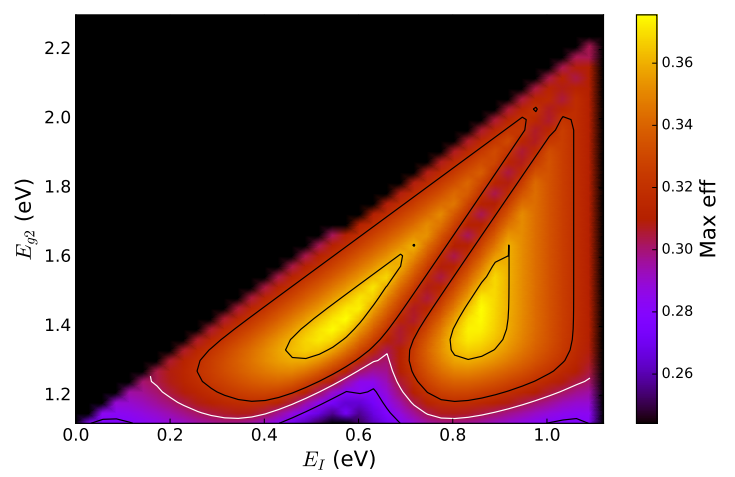

FIG. 4. Maximum ECUC efficiency in detailed balance, with $E_{g 1}=1.12 \mathrm{eV}$ as a function of $E_{g 2}$ and $E_{I}$ at 1 sun concentration. The detailed balance efficiency limit for $E_{g}=1.12 \mathrm{eV}$ is shown with the white contour. Note that the data cutoff at the diagonal (black) occurs because the ECUC requires $E_{I}>E_{g 2}-E_{g 1}$ and $E_{I}<E_{g 1}$.

TABLE II. Maximum detailed balance efficiencies for c-Si $\left(E_{g}=1.12 \mathrm{eV}\right)$ at 1 sun concentration. Note that there is a symmetry for $E_{I}$ mirrored below and above $E_{g 2} / 2$; we take the upper values for $E_{I}$.

\begin{tabular}{ccccc}
\hline System & $E_{g 1}(\mathrm{eV})$ & $E_{g 2}(\mathrm{eV})$ & $E_{I}(\mathrm{eV})$ & Efficiency \\
\hline Single-junction & 1.12 & - & - & $30.2 \%$ \\
IB solar cell & 1.12 & - & 0.85 & $29.7 \%$ \\
ECUC solar cell & 1.12 & 1.47 & 0.86 & $37.4 \%$ \\
\hline
\end{tabular}

for the best-suited $E_{I}$ for an a-Si upconverter on c-Si.

Figure 4 shows the maximum ECUC efficiency with $E_{g 1}=1.12 \mathrm{eV}$ as a function of $E_{g 2}$ and $E_{I}$. The peak efficiencies and band gaps are shown in Table III. The optimal range of $E_{g 2}$ lies approximately between 1.3 and $1.6 \mathrm{eV}$, with the maximum efficiency at $E_{g 2}=1.47 \mathrm{eV}$, with $E_{I}$ near $0.9 \mathrm{eV}$. As $E_{g 2}$ approaches $E_{g 1}$, we recover the IBSC efficiency, which is lower than the ShockleyQueisser limit for a device with $E_{g}=1.12 \mathrm{eV}$. Note that when $E_{g 2}>1.3 \mathrm{eV}$, the ECUC improves efficiencies for all values of $E_{I}$. For a large range of band gaps, it is possible to significantly exceed the SQ limit; therefore, there is potential for high efficiency silicon devices if an ECUC is added.

A promising upconverter material is amorphous silicon, since its band gap of $E_{g 2}=1.55 \mathrm{eV}$ falls in the high-efficiency range [18, and a-Si on c-Si devices are routinely made [19]. Figure 5 shows DB efficiency as a function of $E_{I}$ of a device using c-Si and an a-Si ECUC. All values of $E_{I}$ between $E_{q 2}-E_{g 1}=0.43 \mathrm{eV}$ and $E_{g 1}=1.12 \mathrm{eV}$ give improved efficiencies over the bare c-Si cell. Doping of a-Si is more complicated than in crystalline semiconductors, as dopants can induce local coordination changes and dangling bonds, and the structures vary depending on deposition method 20]. The resulting $E_{I}$ for a dopant in a-Si can thus vary considerably depending on a-Si deposition and dopant precursor

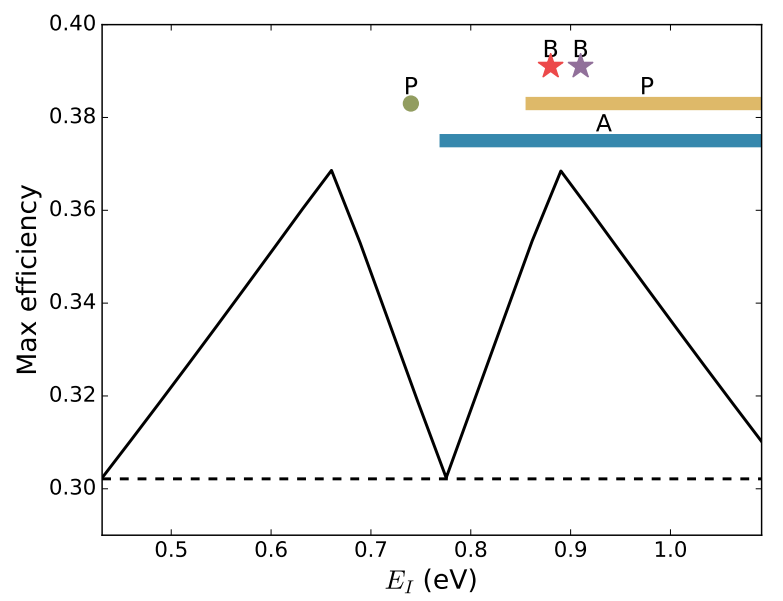

FIG. 5. Maximum ECUC efficiency vs. $E_{I}$ for c-Si $\left(E_{g 1}=\right.$ $1.12 \mathrm{eV})$ and an a-Si upconverter $\left(E_{g 2}=1.55 \mathrm{eV}\right)$. The black dashed line shows the single-junction detailed balance efficiency with $E_{g}=1.12 \mathrm{eV}$. The potential dopants are labelled at their respective $E_{I}$. Doping with $\mathrm{P}$ is shown with the green dot (optical [21]) and a range of values with the yellow line (electrical activation [22]). Thermal activation energies for $\mathrm{B}$ are shown with stars, with red corresponding to doping with $\mathrm{BF}_{3}$ [23] and purple to $\mathrm{B}_{2} \mathrm{H}_{6}$ [21. The blue line shows the range of $E_{I}$ from thermal activation for alkali dopants, including $\mathrm{Na}, \mathrm{K}, \mathrm{Rb}$, and $\mathrm{Cs}$ [24].

and pressure 20]. This variation could allow tuning of ECUC energy levels, which is not generally possible in crystalline semiconductor:dopant materials. To date, devices based on doped a-Si have generally desired shallow dopants, as in c-Si, so the most-studied dopants are those that produce relatively shallow states in the band gap, to give high conductivities. For an ECUC, optically active midgap states are desirable, which is the opposite of the standard case.

Figure 5 also shows estimated energetic positions for some common dopants in a-Si. The most studied dopants include boron and phosphorus as acceptors and donors, respectively, as in c-Si. Even when a-Si has tetrahedrally coordinated silicon, the bond angle distortions tend to make dopant energy levels lie deeper in the gap than in c-Si [25. As an acceptor, B doping using $\mathrm{B}_{2} \mathrm{H}_{6}$ or $\mathrm{BF}_{3}$ gives an electrical activation energy of $E_{I}=0.88-0.91 \mathrm{eV}$, with a higher concentration of active dopant states formed from the $\mathrm{BF}_{3}$ precursor [21, 23. As a donor, $\mathrm{P}$ doping using $\mathrm{PH}_{3}$ gives optical absorption in a band around $E_{g 2}-E_{I}=0.81 \mathrm{eV}$ [21. As can be seen in Fig. 5, this energy level appears close to the middle of the band gap, which allows only minimal improvement in these detailed balance calculations. That dip in efficiency for $E_{I} \approx E_{g 2} / 2$ is an artifact of the nonoverlapping absorption condition, as one of the subgap transitions becomes artificially depleted of photons when $E_{I}$ is close to mid-gap. Removing the non-overlapping absorption requirement, which is only a simplifcation for 
theoretical analysis, reduces the penalty for IB's at midgap [12, 13, so this mid-gap $E_{I}$ can still be beneficial for the ECUC. Doping with $\mathrm{P}$ has also been shown to produce thermal activation energies ranging from $0.74 \mathrm{eV}$ to $0.27 \mathrm{eV}$, depending on concentration of the precursor, with higher activation energies at lower doping concentrations 22]. Alkali atoms as donors, including $\mathrm{Na}, \mathrm{K}$, $\mathrm{Rb}$, and $\mathrm{Cs}$, have been shown to produce thermal activation energies that are similar to each other, ranging from $0.80 \mathrm{eV}$ to $0.20 \mathrm{eV}$, again with higher activation energies at lower dopant concentrations 24]. We interpret these activation energies to be $E_{g 2}-E_{I}$. These values contain overlap with the optimal efficiency range for a c-Si/aSi ECUC. A working ECUC must be optically thick for the subgap photons, which requires either a high dopant concentration or a thick absorber layer. If high dopant concentration is required, the alkali dopant energy levels may be less than than $E_{g 2}-E_{g 1}$ and thus outside of the useful energy range.

The combination of c-Si and a-Si has great potential to make a working ECUC that can improve the efficiency of c-Si solar cells. To realize this potential, the energetic position of those defect states and their optical properties must be characterized, both for the common electrical dopants and possibly a much larger range of potential IB-forming dopants. A wide array of elements may be interesting for a-Si based ECUC, just as a wide array of dopants may be useful for c-Si based IBSC's [26].

\section{CONCLUSIONS}

The ECUC has the potential to improve IB solar cell designs. Its maximum detailed balance efficiency is equal to that of a QRSC, and it may be easier to produce. Though DB calculations do not consider non-radiative processes, they give upper bounds on the efficiency of all photovoltaic devices. At low solar concentration, ECUC has a higher limiting efficiency than IBSC. This effect is realized in the c-Si case at one sun, where an IBSC with non-overlapping absorptions cannot improve on a standard single-gap solar cell, but an ECUC permits significantly improved efficiency. At high concentration, the DB efficiency limits of IBSC, ECUC, and QRSC are all the same, with a significant gain compared to a single junction device. Moving beyond DB, the ECUC architecture allows improved efficiency even with materials having significant nonradiative recombination. It is thus a promising architecture to pursue for near-term development of IB-based devices. The case of a-Si on c-Si provide a promising platform for developing an ECUC with the potential to significantly improve silicon-based solar cell efficiencies.

\section{ACKNOWLEDGMENTS}

We acknowledge helpful conversations with Daniel MacDonald and Wenjie Yang and support from the Natural Sciences and Engineering Research Council of Canada.
[1] William Shockley and Hans J. Queisser, "Detailed Balance Limit of Efficiency of p-n Junction Solar Cells," Journal of Applied Physics 32, 510-519 (1961).

[2] Antonio Luque and Antonio Martí, "Increasing the Efficiency of Ideal Solar Cells by Photon Induced Transitions at Intermediate Levels," Physical Review Letters 78, 5014-5017 (1997).

[3] Y. Okada, N. J. Ekins-Daukes, T. Kita, R. Tamaki, M. Yoshida, A. Pusch, O. Hess, C. C. Phillips, D. J. Farrell, K. Yoshida, N. Ahsan, Y. Shoji, T. Sogabe, and J. F. Guillemoles, "Intermediate band solar cells: Recent progress and future directions," Applied Physics Reviews 2, 1-48 (2015)

[4] M. Yoshida, N. J. Ekins-Daukes, D. J. Farrell, and C. C. Phillips, "Photon ratchet intermediate band solar cells," Applied Physics Letters 100, 3-7 (2012).

[5] Andreas Pusch, Megumi Yoshida, Nicholas P. Hylton, Alexander Mellor, Chris C. Phillips, Ortwin Hess, and Nicholas J. Ekins-Daukes, "Limiting efficiencies for intermediate band solar cells with partial absorptivity: the case for a quantum ratchet," Progress in Photovoltaics: Research and Applications 24, 656-662 (2016).
[6] Andreas Pusch and Nicholas J. Ekins Daukes, "Voltage matching, étendue and ratchet steps in advanced concept solar cells," (2019), arXiv:1906.06793

[7] Anthony Vaquero-Stainer, Megumi Yoshida, Nicholas P. Hylton, Andreas Pusch, Oliver Curtin, Mark Frogley, Thomas Wilson, Edmund Clarke, Kenneth Kennedy, Nicholas J. Ekins-Daukes, Ortwin Hess, and Chris C. Phillips, "Semiconductor nanostructure quantum ratchet for high efficiency solar cells," Communications Physics 1, 2-8 (2018).

[8] Jacob J Krich, Bertrand I Halperin, and Alán AspuruGuzik, "Nonradiative lifetimes in intermediate band photovoltaicsAbsence of lifetime recovery," Journal of Applied Physics 112, 013707 (2012)

[9] Matthew M. Wilkins, Eduard C. Dumitrescu, and Jacob J. Krich, "Minimum material quality threshold for intermediate band solar cells using a multi-band device simulator with fully coupled optics," in IEEE 46th Photovoltaic Specialist Conference (PVSC) (2019) forthcoming.

[10] D. MacDonald, K. McLean, P. N.K. Deenapanray, S. De Wolf, and J. Schmidt, "Electronically-coupled up- 
conversion: An alternative approach to impurity photovoltaics in crystalline silicon," Semiconductor Science and Technology 23, 1-8 (2008)

[11] N. P. Harder and D. Macdonald, "Electronic upconversion: a combination of the advantages of impurity photovoltaics and (optical) up-conversion," in 31st IEEE Photovoltaic Specialists Conference (PVSC) (2005) pp. 110-113.

[12] A Krishna and J J Krich, "Increasing efficiency in intermediate band solar cells with overlapping absorptions," Journal of Optics (United Kingdom) 18, 1-7 (2016)

[13] Lucas Cuadra, Antonio Martí, and Antonio Luque, "Influence of the overlap between the absorption coefficients on the efficiency of the intermediate band solar cell," IEEE Transactions on Electron Devices 51, 1002-1007 (2004)

[14] P Wurfel, "The chemical potential of radiation," Journal of Physics C: Solid State Physics 15, 3967-3985 (1982).

[15] Rune Strandberg and Turid Worren Reenaas, "Limiting efficiency of intermediate band solar cells with spectrally selective reflectors," Applied Physics Letters 97, 031910 (2010)

[16] Antonio Luque and Antonio Martí, "The intermediate band solar cell: Progress toward the realization of an attractive concept," Advanced Materials 22, 160-174 (2010)

[17] Tomah Sogabe, Yasushi Shoji, Mitsuyoshi Ohba, Katsuhisa Yoshida, Ryo Tamaki, Hwen Fen Hong, Chih Hung Wu, Cherng Tsong Kuo, Stanko Tomić, and Yoshitaka Okada, "Intermediate-band dynamics of quantum dots solar cell in concentrator photovoltaic modules," Scientific Reports 4, 1-7 (2014)
[18] David E. Carlson and Christopher R. Wronski, "Amorphous silicon solar cell," Applied Physics Letters 28, 671673 (1976)

[19] Takahiro Mishima, Mikio Taguchi, Hitoshi Sakata, and Eiji Maruyama, "Development status of high-efficiency HIT solar cells," Solar Energy Materials and Solar Cells 95, 18-21 (2011).

[20] D. E. Carlson, "The doping of hydrogenated amorphous silicon and its impact on devices," Critical Reviews in Solid State and Materials Sciences 16, 417-435 (1990).

[21] R. A. Street, D. K. Biegelsen, and R. L. Weisfield, "Recombination in a-Si:H: Transitions through defect states," Physical Review B 30, 5861-5870 (1984).

[22] Akihisa Matsuda, Satoshi Yamasaki, Katsumi Nakagawa, Hideyo Okushi, Kazunobu Tanaka, Sigeru Iizima, Mitsuo Matsumura, and Hideo Yamamoto, "Electrical and Structural Properties of Phosphorous-Doped GlowDischarge Si:F:H and Si:H Films," Japanese Journal of Applied Physics 19, L305-L308 (1980).

[23] A. H. Mahan, Ralph C. Kernsa, and Genevieve Devaud, "BF3-doped amorphous silicon thin films," Journal of Electronic Materials 12, 1033-1049 (1983)

[24] P.G. Le Comber, W.E. Spear, G. Müller, and S. Kalbitzer, "Electrical and photoconductive properties of ion implanted amorphous silicon," Journal of NonCrystalline Solids 35-36, 327-332 (1980)

[25] C. S. Nichols and C. Y. Fong, "Effects of local disorder on donor states in amorphous silicon," Physical Review B 35, 9360-9363 (1987)

[26] Joseph T. Sullivan, Christie B. Simmons, Tonio Buonassisi, and Jacob J. Krich, "Targeted Search for Effective Intermediate Band Solar Cell Materials," IEEE Journal of Photovoltaics 5, 212-218 (2015). 